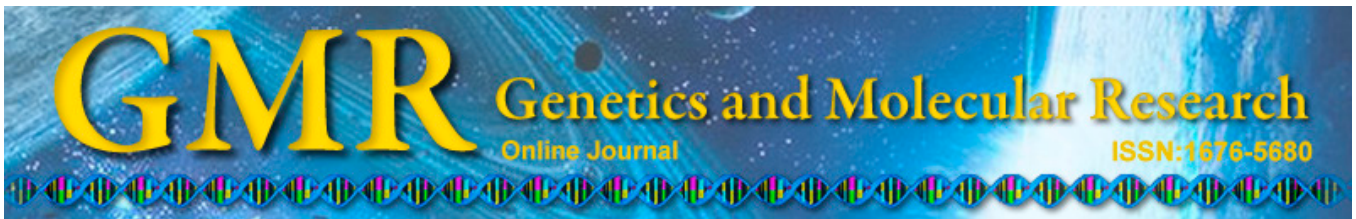

\title{
Expression of high-mobility group box protein 1 in diabetic foot atherogenesis
}

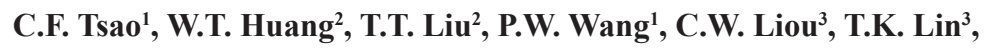 \\ C.J. Hsieh ${ }^{1}$ and S.W. Weng ${ }^{1}$
}

${ }^{1}$ Mitochondrial Research Unit, Department of Internal Medicine, Kaohsiung Chang Gung Memorial Hospital, Chang Gung University College of Medicine, Kaohsiung, Taiwan ${ }^{2}$ Department of Pathology, Kaohsiung Chang Gung Memorial Hospital, Chang Gung University College of Medicine, Kaohsiung, Taiwan

${ }^{3}$ Mitochondrial Research Unit, Department of Neurology, Kaohsiung Chang Gung Memorial Hospital, Chang Gung University College of Medicine, Kaohsiung, Taiwan

Corresponding author: S.W. Weng

E-mail: wenwen@adm.cgmh.org.tw

Genet. Mol. Res. 14 (2): 4521-4531 (2015)

Received June 3, 2014

Accepted October 28, 2014

Published May 4, 2015

DOI http://dx.doi.org/10.4238/2015.May.4.10

\begin{abstract}
The role of high mobility group box 1 (HMGB1) has been demonstrated in stroke and coronary artery disease but not in peripheral arterial occlusive disease (PAOD). The pathogenesis of HMGB1 in acute and chronic vascular injury is also not well understood. We hypothesized that HMGB1 induces inflammatory markers in diabetic PAOD patients. We studied 36 diabetic patients, including 29 patients with PAOD, who had undergone amputation for diabetic foot and 7 nondiabetic patients who had undergone amputation after traumatic injury. Expression of HMGB1 and inflammatory markers were quantified using immunohistochemical staining. Mitochondrial DNA copy number was quantified using real-time polymerase chain reaction. Compared with that in the traumatic amputation group, HMGB1 expression in vessels was significantly higher in the diabetes and diabetic
\end{abstract}


PAOD groups. In all subjects, arterial stenosis grade was positively correlated with the expression levels of HMGB1, 8-hydroxyguanosine, malondialdehyde, vascular cell adhesion molecule 1, and inflammatory markers CD3, and CD68 in both the intima and the media of vessels. Furthermore, HMGB1 expression level was positively correlated with 8-hydroxyguanosine, vascular cell adhesion molecule 1, nuclear factor$\kappa \mathrm{B}, \mathrm{CD} 3$, and CD68 expression. Within the PAOD subgroup, subjects with HMGB1 expression had higher expression of the autophagy marker LC3A/B and higher mitochondrial DNA copy number. HMGB1 may be an inflammatory mediator with roles in oxidative damage and proinflammatory and inflammatory processes in diabetic atherogenesis. Moreover, it may have dual effects by compensating for increased mitochondrial DNA copy number and increased autophagy marker expression.

Key words: Atherosclerosis; Diabetes mellitus; Diabetic foot; High mobility group box 1; Peripheral arterial occlusive disease

\section{INTRODUCTION}

Diabetic foot is among the many complication of diabetes. More than $2 \%$ of community-based diabetic patients develop new foot ulcers each year (Abbott et al., 2002). The incidence of amputation in patients with diabetic foot ulcer is high; in hospitals in Taiwan, more than $94 \%$ of patients treated for diabetic foot had an associated foot infection. The prevalence of in-hospital diabetic foot decreased from 1.68 to $1.02 \%$ from 2000-2009 (P $<0.001)$, but the treatment outcome of lower-extremity amputation did not improve (mean amputation rate: $28.35 \%$ ) (Huang et al., 2012). Thus, a greater understanding of the pathogenesis of diabetic foot and new treatment strategies for this condition are critical.

Peripheral artery disease is one of the most important mechanisms of diabetic foot. The risk of peripheral artery disease is increased in patients with diabetes according to the NHANES study (Selvin and Erlinger, 2004). Moreover, atherosclerosis is a pathologic process that causes coronary, cerebral, and peripheral artery disease (Faxon et al., 2004; Libby et al., 2011). Atherosclerosis involves several highly interrelated processes, including lipid disturbances, platelet activation, thrombosis, endothelial dysfunction, inflammation, oxidative stress, vascular smooth cell activation, altered matrix metabolism, remodeling, and genetic factors (Libby, 2002). Decreased nitric oxide production, increased oxidative stress, and impaired endothelial progenitor cell function are the main mechanisms involved in the accelerated atherosclerotic process observed in type 2 diabetes mellitus (DM) patients (Tousoulis et al., 2012). The main inflammatory markers that provide prognostic information regarding the outcome and progression of type $2 \mathrm{DM}$ include C-reactive protein, tumor necrosis factor$\alpha$, interleukin-6, intercellular adhesion molecule-1, and vascular cell adhesion molecule 1 (VCAM-1) (Tousoulis et al., 2013).

High mobility group box 1 (HMGB1) is a nonhistone DNA-binding protein composed of 215 amino acid residues organized into 3 domains that include 2 tandem HMG box domains (A box and B box) configured in an L shape and a C-terminal tail of 30 amino acids (Tsuda et al., 1988; Read et al., 1993). Recent studies have suggested that HMGB1 induces an inflam- 
matory response during vascular injury. HMGB1 is an endogenous mediator of inflammation and lesion formation via the toll-like receptor 9 pathway in response to vascular injury (Hirataa et al., 2013). HMGB1 also promotes an inflammatory response by inducing the expression of intercellular adhesion molecule-1 and P-selectin via the receptor for advanced glycation end products-mediated stimulation of the endoplasmic reticulum stress pathway (Luo et al., 2013). However, the role of HMGB1 in diabetic patients with peripheral arterial occlusive disease (PAOD) remains unclear. In this study, we examined HMGB1 and its potentially related pathway in the atherosclerotic vessels of patients with diabetic foot to clarify the role of HMGB1 in diabetic atherogenesis.

\section{MATERIAL AND METHODS}

\section{Study subjects}

We recruited 36 patients with type $2 \mathrm{DM}$ and 7 nondiabetic controls. All patients with type 2 DM had undergone leg amputation to treat unhealed leg ulcers. The control patients had undergone leg amputation related to traumatic injuries.

Using pathologic examination, we classified atherosclerosis into 4 groups depending on the level of arterial occlusion: grade 0 was $0 \%$; grade 1 was $0-25 \%$; grade 2 was $25-50 \%$, and grade 3 was more than $50 \%$. The prevalence rates of arterial stenosis from grades $0-3$ in the diabetic group were 19.4 (7/36), 13.9 (5/36), $27.8(10 / 36)$, and 38.9\% $(14 / 36)$, respectively. In contrast, the nondiabetic group showed no arterial stenosis. There were 29 patients with and 14 patients without stenosis. The studies were conducted according to the guidelines of the Declaration of Helsinki, and the protocol for this study was approved by the Ethics Committee of the Chang Gung Memorial Hospital. All subjects provided written informed consent.

\section{Quantification of mitochondrial DNA (mtDNA) copy number with real-time polymerase chain reaction (PCR)}

Relative mtDNA copy numbers were measured using real-time PCR and normalized by simultaneous quantification of nuclear DNA. PCR was performed in an ABI PRISM 7700 sequence detection system (PE Biosystems, Foster City, CA, USA) using a SYBR green PCR master mix kit (Applied Biosystems, Foster City, CA, USA). The forward and reverse primers for $\beta$-actin, the nuclear gene, were 5'-TCACCCACACTGTGC CCATCTAGGA-3' and 5'-CAGCGGAACCGCTCATTGCCAATGG-3', respectively. The forward and reverse primers for ND1, an mtDNA gene sequence, were 5'-TGGGTACA ATGAGGAGTAGG-3' and 5'-GGAGTAATCCAGGTCGGT-3', respectively. PCR was performed for 40 cycles with $10 \mathrm{ng}$ DNA in a $12.5-\mu \mathrm{L}$ reaction mixture using a SYBR green PCR master mix kit and $50 \mathrm{nmol}$ forward and reverse primers. PCR cycling conditions included $15 \mathrm{~s}$ denaturation at $95^{\circ} \mathrm{C}, 20 \mathrm{~s}$ annealing at $60^{\circ} \mathrm{C}$, and $15 \mathrm{~s}$ extension at $72^{\circ} \mathrm{C}$. Melting curve analysis was performed for $20 \mathrm{~min}$ after real-time PCR, and analysis was carried out using the Dissociation Curve Software (Precision Melt Analysis ${ }^{\mathrm{TM}}$ Software-Bio-Rad, USA). Amplified products were denatured and reannealed at different temperatures to detect their specific melting temperatures. The threshold cycle number $(\mathrm{Ct})$ values were determined in the same quantitative PCR run. The results were con- 
firmed by conducting a second run. Ct values were used as a measure of the copy number, and $\mathrm{Ct}$ value differences were used to quantify mtDNA copy number relative to $\beta$-actin, calculated as follows: relative copy number $(\mathrm{Rc})=2^{\Delta \mathrm{Ct}}$, where $\Delta \mathrm{Ct}$ is $\mathrm{Ct}_{\beta a c t i n}-\mathrm{Ct}_{\mathrm{ND1}}$. There was good reproducibility within and between runs.

\section{Immunohistochemical examination}

Immunohistochemical staining was performed using $2-\mu \mathrm{m}$ paraffin-embedded tissue sections on Fisherbrand Superfrost Plus slides (Fisher Scientific, Waltham, MA, USA). Antibodies directed against 8-hydroxyguanosine (8-OHdG, 1:2000; Abcam, Cambridge, UK), malondialdehyde (MDA, 1:1000; Abcam), HMGB1 (1:100; Abcam), LC3A/B (1:50; Abcam), CD68 (1:50; Abcam), CD3 (1:100; Abcam), VCAM-1 (1:200; Abcam), and nuclear factor [(NF-kB p65 (1:2000; Abcam)] were used with the EnVision+ HRP detection system (Dako, Glostrup, Denmark) with an ImmPRESSTM DAB Peroxidase Substrate kit (Burlingame, CA, USA). The percentage of positive staining was determined semiquantitatively using a scale ranging from $0(-)$ to $4(++++)$. Grading with approximate percentages indicating the number of relevant cells showing a positive reaction was as follows: 0$)$ no specific immunohistologic reaction visible; 1$)$ minimal (1-10\% of relevant cells showing a weakly positive reaction; 2 ) mild (up to $25 \%$ showing a strong positive reaction); 3 ) moderate (up to $50 \%$ showing a strong positive reaction); 4) marked (more than 50\% showing a strong positive reaction). Grades 2-4 were defined as positive, and 0 and 1 as negative. All photomicrographs were acquired using an Olympus Insight color camera (Tokyo, Japan).

\section{Statistical analysis}

Logarithmic transformation of data was used because the original values of the relative mtDNA copy number did not show a normal distribution. We used the $t$-test and chisquare analysis of variance for further comparison and analysis. Data are reported as means \pm standard deviation. Relationships between different variables were analyzed using Spearman's correlation. $\mathrm{P}<0.05$ was considered to be statistically significant.

\section{RESULTS}

The differences in immunohistochemistry results for oxidative stress and inflammatory markers in the PAOD and DM groups are shown in Tables 1 and 2, respectively. Subjects with DM and diabetic PAOD showed significantly higher expression of HMGB1 in both the arterial intima and arterial media. Oxidative stress markers in both the intima and media, including 8-OHdg and MDA, were expressed at significantly higher levels in subjects with PAOD. Increased expression of macrophage (CD3) and T-lymphocyte (CD68) markers was also observed in both the DM and PAOD groups. Regarding proinflammatory markers, VCAM-1 expression was significantly higher in the intima in both the DM and PAOD groups, and NF- $\mathrm{BB}$ p65 was slightly significantly higher in the DM group. An autophagy marker, LC3A/B, was expressed at similar levels in the PAOD and nonstenosis groups. 
Table 1. Differences in oxidative stress and inflammatory markers between the PAOD and nonstenosis groups.

\begin{tabular}{|c|c|c|c|c|}
\hline & Intima & & Media & \\
\hline & Arterial stenosis $(\mathrm{N}=29)$ vs nonstenosis $(\mathrm{N}=14)$ & P value & Arterial stenosis $(\mathrm{N}=29)$ vs nonstenosis $(\mathrm{N}=14)$ & $P$ value \\
\hline 8-OHdg & $69 \%(20 / 29)$ vs $35.7 \%(5 / 14)$ & 0.038 & $82.8 \%(24 / 29)$ vs $50.0 \%(7 / 14)$ & 0.025 \\
\hline MDA & $58.6 \%(17 / 29)$ vs $28.6 \%(4 / 14)$ & 0.065 & $62.1 \%(18 / 29)$ vs $21 / 4 \%(3 / 14)$ & 0.012 \\
\hline CD3 & $55.2 \%(16 / 29)$ vs $0 \%(0 / 14)$ & $<0.001$ & $48.3 \%(14 / 29)$ vs $7.1 \%(1 / 14)$ & 0.008 \\
\hline CD68 & $79.3 \%(23 / 29)$ vs $7.1 \%(1 / 14)$ & $<0.001$ & $86 / 2 \%(25 / 29)$ vs $21.4 \%(3 / 14)$ & $<0.001$ \\
\hline VCAM-1 & $89.7 \%(26 / 29)$ vs $50 \%(7 / 14)$ & 0.004 & $93.1 \%(27 / 29)$ vs $85.7 \%(12 / 14)$ & 0.434 \\
\hline $\mathrm{NF}-\kappa \mathrm{B}$ p 65 & $17.2 \%(5 / 29)$ vs $14.3 \%(2 / 14)$ & 0.806 & $31 \%(9 / 29)$ vs $21.4 \%(3 / 14)$ & 0.511 \\
\hline $\mathrm{LC} 3 \mathrm{~A} / \mathrm{B}$ & $3.4 \%(1 / 29)$ vs $0 \%(0 / 14)$ & 0.482 & $20.7 \%(6 / 29)$ vs $28.6 \%(4 / 14)$ & 0.566 \\
\hline HMGB1 & $48.3 \%(14 / 29)$ vs $14.3 \%(2 / 14)$ & 0.031 & $51.7 \%(15 / 29)$ vs $14.3 \%(2 / 14)$ & 0.019 \\
\hline
\end{tabular}

The immunopositivity of the sections was graded by two pathologists using a scale of 0 (-) to 4 (++++). Grading with approximate percentages indicating the numbers of relevant cells showing a positive reaction: (0) no specific immunohistologic reaction visible; (1) minimal: $1-10 \%$ showing a weakly positive reaction; (2) mild: up to $25 \%$ showing a strong positive reaction; (3) moderate: up to 50\% showing a strong positive reaction; (4) marked: more than $50 \%$ showing a strong positive reaction. Grades 2-4 were defined as positive, and grades 0 and 1 were defined as negative. HMGB1, high mobility group box 1; MDA, malondialdehyde; NF- $\kappa \mathrm{B}$, nuclear factor- $\kappa \mathrm{B}$; 8-OHdg, 8-hydroxyguanosine; VCAM-1, vascular cell adhesion molecule 1.

Table 2. Differences in oxidative stress and inflammatory markers between the diabetes mellitus (DM) and nondiabetic groups.

\begin{tabular}{|c|c|c|c|c|}
\hline & Intima & & Media & \\
\hline & $\mathrm{DM}(\mathrm{N}=36) v s$ control $(\mathrm{N}=7)$ & $P$ value & $\mathrm{DM}(\mathrm{N}=36) v s$ control $(\mathrm{N}=7)$ & $P$ value \\
\hline 8 -OHdg & $61.1 \% \%(22 / 36)$ vs $42.9 \%(3 / 7)$ & 0.314 & $75.0 \%(27 / 36)$ vs $57.1 \%(4 / 7)$ & 0.335 \\
\hline MDA & $52.8 \%(19 / 36)$ vs $28.6 \%(2 / 7)$ & 0.412 & $52.8 \%(19 / 36)$ vs $28.6 \%(2 / 7)$ & 0.412 \\
\hline CD3 & $44.4 \%(16 / 36)$ vs $0 \%(0 / 7)$ & 0.035 & $41.7 \%(15 / 36)$ vs $0 \%(0 / 7)$ & 0.077 \\
\hline CD68 & $66.7 \%(24 / 36)$ vs $0 \%(0 / 7)$ & 0.002 & $77.8 \%(28 / 36)$ vs $0 \%(0 / 7)$ & $<0.001$ \\
\hline VCAM-1 & $86.1 \%(31 / 36)$ vs $28.6 \%(2 / 7)$ & 0.001 & $94.4 \%(34 / 36)$ vs $71.4 \%(5 / 7)$ & 0.118 \\
\hline$N F-\kappa B$ p 65 & $19.4 \%(7 / 36)$ vs $0 \%(0 / 7)$ & 0.202 & $33.3 \%(12 / 36)$ vs $0 \%(0 / 7)$ & 0.072 \\
\hline $\mathrm{LC} 3 \mathrm{~A} / \mathrm{B}$ & $2.8 \%(1 / 36)$ vs $0 \%(0 / 7)$ & 0.655 & $19.4 \%(7 / 36)$ vs $42.9 \%(3 / 7)$ & 0.18 \\
\hline HMGB1 & $44.4 \%(16 / 36)$ vs $0 \%(0 / 7)$ & 0.026 & $47.2 \%(17 / 36)$ vs $0 \%(0 / 7)$ & 0.019 \\
\hline
\end{tabular}

The immunopositivity of the sections was graded by two pathologists using a scale of $0(-)$ to $4(++++)$. Grading with approximate percentages indicating the numbers of relevant cells showing a positive reaction: (0) no specific immunohistologic reaction visible; (1) minimal: $1-10 \%$ showing a weakly positive reaction; (2) mild: up to $25 \%$ showing a strong positive reaction; (3) moderate: up to 50\% showing a strong positive reaction; (4) marked: more than $50 \%$ showing a strong positive reaction. Grades $2-4$ were defined as positive, and grades 0 and 1 were defined as negative. HMGB1, high mobility group box 1; MDA, malondialdehyde; NF- $\kappa \mathrm{B}$, nuclear factor- $\kappa \mathrm{B} ; 8-\mathrm{OHdg}$, 8-hydroxyguanosine; VCAM-1, vascular cell adhesion molecule 1.

Table 3 and Figure 1 summarize the correlations between experimental variables in the immunohistochemistry grading of proinflammatory markers, LC3A/B, HMGB1, oxidative stress markers, and the level of arterial stenosis. We found that the severity of arterial stenosis was positively correlated with the expression of oxidative stress markers (8-OHdG and MDA), the proinflammatory marker (VCAM-1), inflammatory markers (CD3 and CD68), and HMGB1 in both the intima and media of vessels. HMGB1 expression level was positively correlated with that of proinflammatory markers (VCAM-1 and NF- $\kappa \mathrm{B}$ ) and inflammatory markers (CD3 and CD68) in both the intima and the media as well. HMGB1 was also positively correlated with 8-OHdG expression and marginally significantly correlated with LC3A/B expression in the media of vessels. Within the DM subgroup, HMGB1 expression in the media was significantly correlated with high LC3A/B expression in the media $(\mathrm{r}=0.417 ; \mathrm{P}=0.011)$. 
The correlation between HMGB1 and LC3A/B expression in the media was borderline significant within the PAOD subgroup $(\mathrm{r}=0.346 ; \mathrm{P}=0.066)$.

Table 3. Correlation between PAOD and atherosclerosis-related factors.

\begin{tabular}{|c|c|c|c|c|c|c|c|c|}
\hline $\begin{array}{l}\text { Location } \\
\text { All subjects }(\mathrm{N}=43)\end{array}$ & $\begin{array}{c}\text { Intima } \\
\text { Arterial stenosis } \\
\mathrm{r}\end{array}$ & $P$ value & $\begin{array}{c}\text { HMGB1 } \\
\text { r }\end{array}$ & $P$ value & $\begin{array}{c}\text { Media } \\
\text { Arterial stenosis } \\
\mathrm{r}\end{array}$ & $\mathrm{P}$ value & $\begin{array}{c}\text { HMGB1 } \\
\text { r }\end{array}$ & $\mathrm{P}$ value \\
\hline mtDNA copy number & -0.387 & 0.01 & 0.457 & 0.002 & -0.387 & 0.01 & 0.296 & 0.054 \\
\hline 8-OHdg & 0.372 & 0.014 & 0.273 & 0.077 & 0.339 & 0.026 & 0.322 & 0.035 \\
\hline MDA & 0.616 & $<0.001$ & 0.218 & 0.161 & 0.628 & $<0.001$ & 0.207 & 0.183 \\
\hline CD3 & 0.689 & $<0.001$ & 0.341 & 0.025 & 0.555 & $<0.001$ & 0.352 & 0.021 \\
\hline CD68 & 0.682 & $<0.001$ & 0.336 & 0.028 & 0.551 & $<0.001$ & 0.455 & 0.002 \\
\hline VCAM-1 & 0.51 & $<0.001$ & 0.475 & 0.001 & 0.316 & 0.039 & 0.481 & 0.001 \\
\hline NF-кB p 65 & 0.294 & 0.056 & 0.554 & $<0.001$ & 0.069 & 0.66 & 0.559 & $<0.001$ \\
\hline $\mathrm{LC} 3 \mathrm{~A} / \mathrm{B}$ & 0.105 & 0.503 & 0.232 & 0.134 & -0.092 & 0.558 & 0.282 & 0.067 \\
\hline HMGB1 & 0.457 & 0.002 & - & - & 0.296 & 0.054 & - & - \\
\hline
\end{tabular}

HMGB1, high mobility group box 1; MDA, malondialdehyde; mtDNA, mitochondrial DNA; NF- $\kappa B$, nuclear factor-kB; 8-OHdg, 8-hydroxyguanosine; VCAM-1, vascular cell adhesion molecule 1.

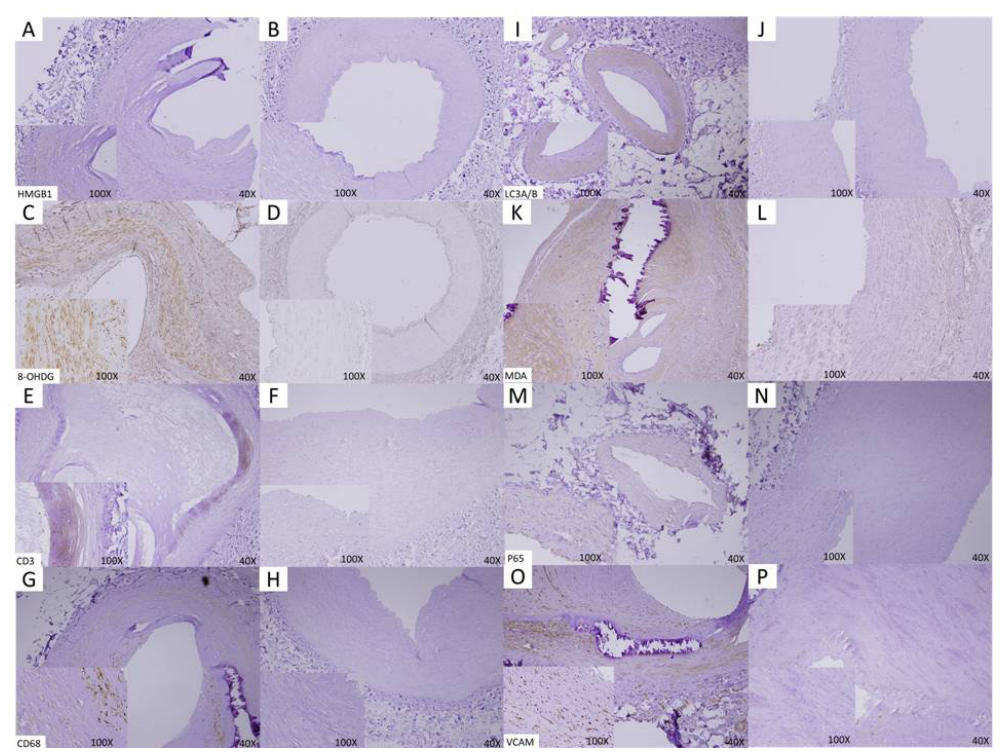

Figure 1. Immunohistochemical studies for HMGB1 showed positive staining in arteries with atherosclerotic change (A) compared with negative staining in normal arteries (B). Notably, a positive correlation was observed between HMGB1, atherosclerosis, and 8-OHDG (C), CD3 (E), CD68 (G), LC3A/B (I), malondialdehyde (MDA; $\mathrm{K}), \mathrm{NF}-\kappa \mathrm{B}$ p65 (M), and VCAM-1 (O). On the contrary, all markers revealed negative staining in arteries $(\mathbf{D}, \mathbf{F}$, $\mathbf{H}, \mathbf{J}, \mathbf{K}, \mathbf{L}, \mathbf{N}, \mathbf{P})$.

Comparisons between subjects with positive and negative expression of HMGB1 are shown in Table 4 and Figure 1. These results are similar to those of grading correlation analysis between HMGB1 and other vascular injury markers as shown in Table 3. Subjects with HMGB1 expression showed higher expression of CD68 and NF-kB p65 in both the intima and the media and higher expression of VCAM-1 in the intima only was statistically significant. Within the PAOD subgroup, subjects with HMGB1 expression showed significantly higher expression of 
NF-кB p65 and higher expression of LC3A/B in the media; the difference was slightly statistically significant. In the intima, higher expression of VCAM-1 was also marginally significant.

\begin{tabular}{|c|c|c|c|c|}
\hline & HMGB1 IHC expression & Negative & Positive & \\
\hline Location & All subjects $(\mathrm{N}=43)$ & & & P value \\
\hline Intima & CD68 & $44.4 \%(12 / 27)$ & $75 \%(12 / 16)$ & 0.051 \\
\hline Media & CD68 & $50 \%(13 / 26)$ & $88.2 \%(15 / 17)$ & 0.01 \\
\hline Intima & VCAM-1 & $63 \%(17 / 27)$ & $100 \%(16 / 16)$ & 0.005 \\
\hline Intima & NF-кB p 65 & $7.4 \%(2 / 27)$ & $31.3 \%(5 / 16)$ & 0.041 \\
\hline Media & NF-кB p 65 & $7.7 \%(2 / 26)$ & $58.8 \%(10 / 17)$ & $<0.001$ \\
\hline Location & $\begin{array}{l}\text { HMGB1 IHC expression } \\
\text { Stenosis subgroup }(\mathrm{N}=29)\end{array}$ & Negative & Positive & $P$ value \\
\hline Intima & VCAM-1 & $80 \%(12 / 15)$ & $100 \%(14 / 14)$ & 0.077 \\
\hline Intima & MnSOD & $6.7 \%(1 / 15)$ & $35.7 \%(5 / 14)$ & 0.054 \\
\hline Media & NF-кB p 65 & $7.1 \%(1 / 14)$ & $53.3 \%(8 / 15)$ & 0.007 \\
\hline Media & $\mathrm{LC} 3 \mathrm{~A} / \mathrm{B}$ & $7.1 \%(1 / 14)$ & $33.3 \%(5 / 15)$ & 0.082 \\
\hline
\end{tabular}

HMGB1, high mobility group box 1; IHC, immunohistochemical; MDA, malondialdehyde; MnSOD, manganese superoxide dismutase; mtDNA, mitochondrial DNA; NF- $\mathrm{B}$, nuclear factor- $\kappa \mathrm{B}$; 8-OHdg, 8-hydroxyguanosine; VCAM-1, vascular cell adhesion molecule 1.

We previously found that compared with nondiabetic patients, diabetic patients had significantly fewer copies of mtDNA $(2.55 \pm 0.15$ vs $2.73 \pm 0.16, \mathrm{P}=0.008)$. Compared to patients without arterial stenosis, patients with PAOD also had significantly fewer copies of mtDNA $(2.54 \pm 0.13$ vs $2.66 \pm 0.20, \mathrm{P}=0.035)$. We found that HMGB1 also plays a role in compensating for the lower mtDNA copy number in the PAOD group. Within the PAOD subgroup, subjects expressing HMGB1 in either the intima or the media had significantly higher mtDNA copy numbers $(2.604 \pm 0.136$ vs $2.485 \pm 0.104$ and $2.608 \pm 0.131$ vs $2.472 \pm 0.096$; $\mathrm{P}=0.013$ and 0.004 , respectively). Within the DM subgroup, a similar trend was observed in subjects expressing HMGB1 in the media $(2.601 \pm 0.128$ vs $2.505 \pm 0.163 ; \mathrm{P}=0.061)$.

\section{DISCUSSION}

Peripheral artery disease is a risk factor for diabetic foot in patients with diabetes. Understanding the pathophysiology of peripheral artery disease may offer insight into new methods for preventing diabetic foot. Previous studies revealed that macrophages are a major source of HMGB1 in human atherosclerotic lesions. Upregulation and secretion of HMGB1 can amplify inflammatory responses and may also contribute to macrophage accumulation, thereby promoting atherogenesis (Kalinina et al., 2004). Fiuza et al. (2002) found that recombinant human HMGB1 increases expression of intercellular adhesion molecule-1, VCAM1, and the receptor for advanced glycation end products. HMGB1 elicits proinflammatory responses in endothelial cells and may contribute to alterations in endothelial cell function. However, the role of HMGB1 in diabetic foot patients with PAOD remains unclear. The results of our study showed that HMGB1 expression was higher in patients with both diabetic foot and arterial stenosis. In addition, the correlations between HMGB1 and proinflammatory markers VCAM-1 and NF- $\kappa B$ in either the intima or the media of vessels were significantly positive, indicating that the HMGB1-induced proinflammation response may play a role in the pathogenesis of diabetic foot atherogenesis. 
Studies in animal models have suggested that HMGB1 plays a pathophysiological role in atherosclerosis by exerting proatherogenic effects by stimulating macrophage migration, modulating proinflammatory mediators, and accumulating immune and smooth muscle cells. Furthermore, HMGB1 neutralization reduces diet-induced atherosclerosis in apolipoprotein E-deficient mice (Kanellakis et al., 2011). Concordant with previous findings in animal models, the results of the present study in humans showed positive correlations between CD68 (a macrophage marker), CD3 (a T-cell marker), VCAM-1, and NF- $\mathrm{BB}$ (proinflammatory markers), and HMGB1 in both the intima and the media of vessels, indicating that HMGB1 neutralization may be useful for treating PAOD in patients with diabetic foot disease.

Acute coronary syndrome and ischemic stroke are associated with atherosclerosis, particularly in patients with diabetes. Serum HMGB1 levels are significantly elevated in both myocardial ischemia patients and cerebral ischemia patients (Goldstein et al., 2006). In the present study, we demonstrated that HMGB1 expression was greater in the atherosclerotic vessels of the target organs of PAOD patients. HMGB1 is a proinflammatory cytokine derived from both injured endothelium and activated macrophages/monocytes. Increased serum HMGB1 level is associated with coronary artery disease in both nondiabetic and type $2 \mathrm{DM}$ patients (Yan et al., 2009), and the degree of serum HMGB1 increase is correlated with the severity of coronary artery stenosis (Hu et al., 2009). Serum HMGB1 is also elevated and induces inflammatory reactions in cerebral ischemia (Vogelgesang et al., 2010).

However, HMGB1 may have dual effects after ischemic damage, and HMGB1 levels peak twice during hypoxemia, during the acute and the late phases. In 2005, Limana et al. (2005) observed significant recovery of cardiac performance in HMGB1-treated mice. Recently, postischemic treatment with HMGB1 was shown to significantly improve myocardial functional recovery after global ischemia/reperfusion in association with decreased infarct size (Abarbanell et al., 2011). In experimental studies of ischemic stroke, HMGB1 mediated beneficial plasticity and recovery in many cells of the neurovascular unit during the delayed post-stroke phase (Hayakawa et al., 2010b). Hayakawa et al. (2010a) demonstrated that a significant decrease in HMGB1-positive reactive astrocytes worsened behavioral recovery. In summary, among patients with coronary artery disease or cerebral ischemic disease, HMGB1 may have dual effects by acting as a proinflammatory mediator in the acute phase and being involved in recovery and remodeling during the late phase. However, the mechanisms of these dual effects of HMGB1 in atherosclerotic disease remain unclear.

In our previous study, we found that diabetic atherogenesis is associated with decreased levels of mtDNA (Chien et al., 2012). The results of the present study showed that although HMGB1 may act as an inflammatory mediator based on its positive correlation with the expression of oxidative stress markers (8-OHdG and MDA), a proinflammatory marker (VCAM-1), and inflammatory markers (CD3 and CD68) both in the intima and the media of vessels, an HMGB1-related compensation mechanism may also function in diabetic patients with PAOD. Patients with higher HMGB1 expression in the PAOD and DM subgroups showed higher mtDNA copy numbers and higher expression of the autophagy marker LC3A/B. However, the compensated mtDNA copy number was still lower than the number in the nonstenosis and nondiabetic subgroups. Furthermore, many studies have confirmed HMGB1-regulated autophagy, especially after oxidative damage (Tang et al., 2010; Induces, 2011; Livesey et al., 2012). Severe autophagy deficiency in insulin target tissues can induce the mitochondrial stress response, and dysregulation of autophagy may contribute to the development of metabolic disorders, including insulin resistance, DM, obesity, and atherosclerosis (Kim and Lee, 2014). Deficiencies 
in autophagy maturation may result in an imbalance of arterial smooth muscle cell homeostasis and thus may be an important atherogenic mechanism in coronary arteries (Kim et al., 2013). In the present study, HMGB1-related LC3A/B expression in the media of atherosclerotic vessels appeared to play a compensatory role in diabetic foot patients with PAOD. However, the roles of HMGB1 in the causal and responsive mechanisms of diabetic foot atherogenesis remain unclear.

Several studies have examined the role of HMGB1 intervention in atherosclerotic diseases. A 2007 study showed that anti-HMGB1 monoclonal antibody inhibited the increased permeability of the blood-brain barrier, activation of microglia, and expression of tumor necrosis factor- $\alpha$ and inducible nitric oxide synthase and suppressed the activity of matrix metallopeptidase 9. Intravenous injection of neutralizing anti-HMGB1 monoclonal antibody is a novel therapeutic strategy for treating ischemic stroke (Liu et al., 2007). Zhang et al. (2011) also found that anti-HMGB1 monoclonal antibody effectively treats brain ischemia by inhibiting the development of brain edema via protection of the blood-brain barrier and efficient clearance of circulating HMGB1. Furthermore, Abarbanell et al. (2011) observed a dose-dependent effect of HMGB1 on myocardial recovery after acute global ischemia/reperfusion injury in rat hearts. In addition, HMGB1 induces a proangiogenic phenotype in endothelial cells in vivo and triggers a potent angiogenic response (Mitola et al., 2006; van Beijnum et al., 2006; Kitahara et al., 2008), suggesting that HMGB1 may play an important role in the repair of ischemic damage. Recent studies have also found that endogenous HMGB1 is crucial for ischemia-induced angiogenesis in diabetic mice and that HMGB1 protein administration enhances collateral blood flow in the ischemic hind limbs of diabetic mice in a vascular endothelial growth factor-dependent manner (Biscetti et al., 2010). Although the mechanism of HMBG1 during the early and late phases of coronary artery disease and cerebral ischemic disease remains unexamined, manipulation of HMGB1 may provide a new treatment strategy for diabetic foot in patients with PAOD.

In conclusion, our data revealed that HMGB1 may be an inflammatory mediator related to oxidative damage and proinflammatory and inflammatory processes in diabetic atherogenesis. HMGB1 may have dual effects by compensating for increased mtDNA copy number and increased expression of autophagy markers.

\section{ACKNOWLEDGMENTS}

Research supported by the National Science Council, Executive Yuan (ROC; \#NSC98-2314-B-182A-017-MY2).

\section{REFERENCES}

Abarbanell AM, Hartley JA, Herrmann JL, Weil BR, et al. (2011). Exogenous high-mobility group box 1 improves myocardial recovery after acute global ischemia/reperfusion injury. Surgery 149: 329-335.

Abbott CA, Carrington AL, Ashe H, Bath S, et al. (2002). The North-West Diabetes Foot Care Study: incidence of, and risk factors for, new diabetic foot ulceration in a community-based patient cohort. Diabet. Med. 19: 377-384.

Biscetti F, Straface G, De Cristofaro R, Lancellotti S, et al. (2010). High-mobility group box-1 protein promotes angiogenesis after peripheral ischemia in diabetic mice through a VEGF-dependent mechanism. Diabetes 59: 14961505.

Chien MC, Huang WT, Wang PW, Liou CW, et al. (2012). Role of mitochondrial DNA variants and copy number in diabetic atherogenesis. Genet. Mol. Res. 11: 3339-3348. 
Faxon DP, Fuster V, Libby P, Beckman JA, et al. (2004). Atherosclerotic Vascular Disease Conference: Writing Group III: pathophysiology. Circulation 109: 2617-2625.

Fiuza C, Bustin M, Talwar S, Tropea M, et al. (2002). Inflammation-promoting activity of HMGB1 on human microvascular endothelial cells. Blood 101: 2652-2660.

Goldstein RS, Gallowitsch-Puerta M, Yang L, Rosas-Ballina M, et al. (2006). Elevated high-mobility group box 1 levels in patients with cerebral and myocardial ischemia. Shock 25: 571-574.

Hayakawa K, Nakano T, Irie K, Higuchi S, et al. (2010a). Inhibition of reactive astrocytes with fluorocitrate retards neurovascular remodeling and recovery after focal cerebral ischemia in mice. J. Cereb. Blood Flow Metab. 30: 871882.

Hayakawa K, Qiu J and Lo H (2010b). Biphasic actions of HMGB1 signaling in inflammation and recovery after stroke. Ann. N Y Acad. Sci. 1207: 50-57.

Hirataa Y, Kurobec H, Higashidad M, Fukudae D, et al. (2013). HMGB1 plays a critical role in vascular inflammation and lesion formation via toll-like receptor 9. Atherosclerosis 231: 227-233.

Hu X, Jiang H, Bai Q, Zhou X, et al. (2009). Increased serum HMGB1 is related to the severity of coronary artery stenosis. Clin. Chim. Acta 406: 139-142.

Huang YY, Linb KD, Jiang YD, Chang CH, et al. (2012). Diabetes-related kidney, eye, and foot disease in Taiwan: an analysis of the nationwide data for 2000-2009. J. Formos Med. Assoc. 111: 637-644.

Induces OS (2011). HMGB1 as an autophagy sensor in oxidative stress. Autophagy 7: 904-906.

Kalinina N, Agrotis A, Antropova Y, DiVitto G, et al. (2004). Increased expression of the DNA-binding cytokine HMGB1 in human atherosclerotic lesions: role of activated macrophages and cytokines. Arterioscler. Thromb. Vasc. Biol. 24: 2320-2325.

Kanellakis P, Agrotis A, Kyaw TS, Koulis C, et al. (2011). High-mobility group box protein 1 neutralization reduces development of diet-induced atherosclerosis in apolipoprotein e-deficient mice. Arterioscler. Throm. Vasc. Biol. 31: 313-319.

Kim KH and Lee MS (2014). Autophagy - a key player in cellular and body metabolism. Nat. Rev. Endocrinol. 10: 322-337.

Kim KH, Jeong YT, Oh H, Kim SH, et al. (2013). Autophagy deficiency leads to protection from obesity and insulin resistance by inducing Fgf21 as a mitokine. Nat. Med. 19: 83-92.

Kitahara T, Takeishi Y, Harada M, Niizeki T, et al. (2008). High-mobility group box 1 restores cardiac function after myocardial infarction in transgenic mice. Cardiovasc. Res. 80: 40-46.

Libby P (2002). Inflammation in atherosclerosis. Nature 420: 868-874.

Libby P, Ridker PM and Hansson GK (2011). Progress and challenges in translating the biology of atherosclerosis. Nature 473: 317-325.

Limana F, Germani A, Zacheo A, Kajstura J, et al. (2005). Exogenous high-mobility group box 1 protein induces myocardial regeneration after infarction via enhanced cardiac C-kit+ cell proliferation and differentiation. Circ. Res. 97: e73-e83.

Liu K, Mori S, Takahashi HK, Takahashi, et al. (2007). Anti-high mobility group box 1 monoclonal antibody ameliorates brain infarction induced by transient ischemia in rats. FASEB J. 21:3904-3916.

Livesey KM, Kang R, Vernon P, Buchser W, et al. (2012). p53/HMGB1 complexes regulate autophagy and apoptosis. Cancer Res. 72: 1996-2005.

Luo Y, Lib SJ, Yanga J, Qiuc YZ, et al. (2013). HMGB1 induces an inflammatory response in endothelial cells via the RAGE-dependent endoplasmic reticulum stress pathway. Biochem. Biophys. Res. Commun. 438: 732-738.

Mitola S, Belleri M, Urbinati C, Coltrini D, et al. (2006). Cutting edge: extracellular high mobility group box-1 protein is a proangiogenic cytokine. J. Immunol. 176: 12-15.

Read CM, Cary PD, Crane-Robinson C, Driscoll PC, et al. (1993). Solution structure of a DNA-binding domain from HMG1. Nucleic Acids Res. 21: 3427-3436.

Selvin E and Erlinger TP (2004). Prevalence of and risk factors for peripheral arterial disease in the United States: results from the National Health and Nutrition Examination Survey, 1999-2000. Circulation 110: 738-743.

Tang D, Kang R, Livesey KM, Cheh C-W, et al. (2010). Endogenous HMGB1 regulates autophagy. J. Cell Biol. 190: 881-892.

Tousoulis D, Kampoli AM and Stefanadis C (2012). Diabetes mellitus and vascular endothelial dysfunction: current perspectives. Curr. Vasc. Pharmacol. 10: 19-32.

Tousoulis D, Papageorgiou N, Androulakis E, Siasos G, et al. (2013). Diabetes mellitus-associated vascular impairment: novel circulating biomarkers and therapeutic approaches. J. Am. Coll. Cardiol. 62: 667-676.

Tsuda K, Kikuchi M, Mori K, Waga S, et al. (1988). Primary structure of non-histone protein HMG1 revealed by the nucleotide sequence. Biochemistry 27: 6159-6163.

van Beijnum JR, Dings RP, van der Linden E, Zwaans BM, et al. (2006). Gene expression of tumor angiogenesis dissected: specific targeting of colon cancer angiogenic vasculature. Blood 108: 2339-2348. 
Vogelgesang A, May VE, Grunwald U, Bakkeboe M, et al. (2010). Functional status of peripheral blood T-cells in ischemic stroke patients. PLoS One 5: e8718.

Yan XX, Lu L, Peng WH, Wang LJ, et al. (2009). Increased serum HMGB1 level is associated with coronary artery disease in nondiabetic and type 2 diabetic patients. Atherosclerosis 205: 544-548.

Zhang J, Takahashi HK, Liu K, Wake H, et al. (2011). Anti-high mobility group box-1 monoclonal antibody protects the blood-brain barrier from ischemia-induced disruption in rats. Stroke 42: 1420-1428. 